\title{
ON COMPLETE CONVERGENCE FOR RANDOMLY INDEXED SUMS FOR A CASE WITHOUT IDENTICAL DISTRIBUTIONS
}

\author{
ANNA KUCZMASZEWSKA \\ Technical University of Lublin, Department of Applied Mathematics, \\ Bernardyńska 13, \\ 20-950 Lublin, Poland \\ DOMINIK SZYNAL \\ Institute of Mathematics, UMCS \\ Plac Marii Curie-Skłodowskiej 1, \\ 20-031 Lublin, Poland \\ (Received December 26, 1995 and in revised form August 10, 1996)
}

\begin{abstract}
In this note we extend the complete convergence for randomly indexed sums given by Klesov (1989) to nonidentical distributed random variables.
\end{abstract}

KEY WORDS AND PHRASES: complete convergence, random indexed sums, regular cover, array of rowwise independent random variables.

1991 AMS SUBJECT CLASSIFICATION CODES: 60F15, 60B 12.

\section{INTRODUCTION AND PRELIMINARIES}

The following concept of complete convergence was given by Hsu and Robbins [1].

DEFINITION 1.1. A sequence $\left\{X_{n}, n \geq 1\right\}$ of random variables converges completly to the constant $C$ if

$$
\sum_{n=1}^{\infty} P\left[\left|X_{n}-C\right| \geq \varepsilon\right]<\infty, \quad \forall \varepsilon>0 .
$$

The main result of Hsu and Robbins [1] states that for a sequence $\left\{X_{n}, n \geq 1\right\}$ of i.i.d. random variables with zero expectation and $E X_{1}^{2}<\infty$, we have

$$
\sum_{n=1}^{\infty} P\left[\left|S_{n}\right| \geq n \varepsilon\right]<\infty, \quad \forall \varepsilon>0,
$$

where $S_{n}=\sum_{k=1}^{n} X_{k}$, i.e. the sequence of arithmetic means $S_{n} / n, n \geq 1$, completly convergence to 0 . Erdös [2] proved the converse statement.

Extensions and generalizations of those results were summarized by A. Gut [3]. Extensions of (1.1) to randomly indexed sums of i.i.d. random variables one can find in Szynal [4], Gut 
[5], Zhidong and Chun [6], Adler [7] and Klesov [8]. Some results concerning complete convergence for randomly indexed sums of nonidenticaly distributed random variables were given by Kuczmaszewska and Szynal [9], [10].

In this note we extend results on the complete convergence for randomly indexed sums in spirit of Gut [5] and Klesov [8] to nonidentical distributed random variables.

We use the following concept of regular cover of (the distribution of ) a random variable.

DEFINITION 1.2. (See Pruss [11]). Let $X_{1}, \ldots, X_{n}$ be random variables and let $\xi$ be a random variable possible defined on a different probability space. Then $X_{1}, \ldots, X_{n}$ are said to be a regular cover of (the distribution of) $\xi$ provided we have

$$
E[G(\xi)]=\frac{1}{n} \sum_{k=1}^{n} E\left[G\left(X_{k}\right)\right]
$$

for any measurable function $G$ for which both sides make sense. If $X_{1}, \ldots, X_{n}$ are in addition independent, then we say they form an independent regular cover of $\xi$.

\section{RESULTS.}

The following theorem contains as a particular case the main result of Klesov [8].

THEOREM 2.1. Let $\left\{X_{n k}, n \geq 1, k \geq 1\right\}$ be an array of rowwise independent random variables with $E X_{n k}=0, E\left|X_{n k}\right|^{r}<\infty$, for some $r \geq 1$, and $n \geq 1, k \geq 1$, such that $X_{n 1}, X_{n 2}, \ldots, X_{n k}, n \geq 1, k \geq 1$, form an independent regular cover of a random variable $\xi$ with $E \xi=0, E|\xi|^{r}<\infty$, for some $r \geq 1$. Suppose that $\left\{\nu_{k}, k \geq 1\right\}$ is a sequence of positive integer-valued random variables. Then for $S_{\nu_{n}}=\sum_{k=1}^{\nu_{n}} X_{n k}$ we have:

$$
\sum_{n=1}^{\infty} n^{\alpha r-2} P\left[\left|S_{\nu_{n}}\right| \geq \varepsilon \nu_{n}^{\alpha}\right]<\infty, \quad \forall \varepsilon>0,
$$

for $\alpha>1 / 2, \alpha r>1$ and $\beta \geq 1$, whenever

$$
\sum_{n=1}^{\infty} n^{\alpha r-2} P\left[\nu_{n}<n^{\beta}\right]<\infty,
$$

and (2.1) holds true for $\alpha>1 / 2, \alpha r>1$, and $0<\beta<1$, whenever additionally with (2.2) the condition

$$
\sum_{n=1}^{\infty} n^{\alpha r-2} P\left[\max _{k \leq \nu_{n}}\left|X_{n k}\right| \geq \varepsilon \nu_{n}^{\alpha}\right]<\infty, \quad \forall \varepsilon>0,
$$

is satisfied.

PROOF. Firstly we prove that (2.2) and (2.3) with $\alpha>\frac{1}{2}, \alpha r>1$, and $\beta>0$ imply (2.1). Taking into account

$$
\sum_{n=1}^{\infty} n^{\alpha r-2} P\left[\left|S_{\nu_{n}}\right| \geq \varepsilon \nu_{n}^{\alpha}\right] \leq \sum_{n=1}^{\infty} n^{\alpha r-2} P\left[\left|S_{\nu_{n}}\right| \geq \varepsilon \nu_{n}^{\alpha}, \nu_{n} \geq n^{\beta}\right]+\sum_{n=1}^{\infty} n^{\alpha r-2} P\left[\nu_{n}<n^{\beta}\right]
$$

we see that we need only to show that

$$
\sum_{n=1}^{\infty} n^{\alpha r-2} P\left[\left|S_{\nu_{n}}\right| \geq \varepsilon \nu_{n}^{\alpha}, \nu_{n} \geq n^{\beta}\right]<\infty
$$


Let $\delta>\frac{(\alpha r-1)}{\beta}, \frac{1}{(\alpha r)}<\gamma<1$ and $q$ be a positive integer such that $q>\frac{(1+\delta)}{(\alpha r-1)}$. Define the sets (cf. Klesov [8]):

$$
\begin{aligned}
& B_{n}^{(1)}=\left[\exists k \leq \nu_{n}:\left|X_{n k}\right| \geq \frac{\varepsilon \nu_{n}^{\alpha}}{q}\right], \\
& B_{n}^{(2)}=\left[\exists \text { at least } q \text { indices } k \leq \nu_{n}:\left|X_{n k}\right| \geq \nu_{n}^{\gamma \alpha}\right], \\
& B_{n}^{(3)}=\left[\left|\sum_{k \leq \nu_{n}} X_{n k} I\left[\left|X_{n k}\right|<\nu_{n}^{\gamma \alpha}\right]\right| \geq \frac{\varepsilon \nu_{n}^{\alpha}}{q}\right],
\end{aligned}
$$

where $I[A]$ is the indicator function of an event A. Taking into account that

$$
\left[\left|S_{\nu_{n}}\right| \geq \varepsilon \nu_{n}^{\alpha}\right] \subseteq B_{n}^{(1)} \cup B_{n}^{(2)} \cup B_{n}^{(3)}
$$

we note that (2.4) will be proved if we show that

$$
\sum_{n=1}^{\infty} n^{\alpha r-2} P\left[B_{n}^{(i)} \cap\left[\nu_{n} \geq n^{\beta}\right]\right]<\infty, \quad i=1,2,3 .
$$

For $i=1$ we have

$$
\begin{gathered}
\sum_{n=1}^{\infty} n^{\alpha r-2} P\left[B_{n}^{(1)} \cap\left[\nu_{n} \geq n^{\beta}\right]\right] \leq \sum_{n=1}^{\infty} n^{\alpha r-2} P\left[\exists k \leq \nu_{n}:\left|X_{n k}\right| \geq\left(\varepsilon \nu_{n}^{\alpha}\right) / q\right] \\
\leq \sum_{n=1}^{\infty} n^{\alpha r-2} P\left[\max _{k \leq \nu_{n}}\left|X_{n k}\right| \geq \varepsilon^{\prime} \nu_{n}^{\alpha}\right], \quad \varepsilon^{\prime}=\varepsilon / q .
\end{gathered}
$$

In the case $i=2$ we state that

$$
\begin{gathered}
\sum_{n=1}^{\infty} n^{\alpha r-2} P\left[B_{n}^{(2)} \cap\left[\nu_{n} \geq n^{\beta}\right]\right] \\
\leq \sum_{n=1}^{\infty} n^{\alpha r-2} \sum_{j=1}^{\infty} P\left[B_{n}^{(2)} \cap\left[\nu_{n}=j\right], \nu_{n} \geq n^{\beta}\right] \\
\leq \sum_{n=1}^{\infty} n^{\alpha r-2} \sum_{j=1}^{\infty} \sum_{1 \leq k_{1} \leq k_{2} \leq \ldots \leq k_{q} \leq j} P\left[\nu_{n}=j,\left|X_{n 1}\right| \geq j^{\gamma \alpha}, \ldots,\left|X_{n k_{q}}\right| \geq j^{\gamma \alpha}, \nu_{n} \geq n^{\beta}\right] \\
\leq \sum_{n=1}^{\infty} n^{\alpha r-2-\beta \delta} \sum_{j=1}^{\infty} j^{\delta-q r \gamma \alpha} \sum_{1 \leq k_{1} \leq k_{2} \leq \ldots \leq k_{q} \leq j} E\left|X_{n 1}\right|^{r} \ldots E\left|X_{n k_{q}}\right|^{r} I\left[\nu_{n}=j, \nu_{n} \geq n^{\beta}\right] \\
\leq \sum_{n=1}^{\infty} n^{\alpha r-2-\beta \delta} \sum_{j=1}^{\infty} j^{\delta-q r \gamma \alpha} \sum_{1 \leq k_{1} \leq k_{2} \leq \ldots \leq k_{q} \leq j} E\left|X_{n 1}\right|^{r} \ldots E\left|X_{n k_{q}}\right|^{r} \\
=\sum_{n=1}^{\infty} n^{\alpha r-2-\beta \delta} \sum_{j=1}^{\infty} j^{\delta-q r \gamma \alpha} \sum_{k_{q}=q}^{j} E\left|X_{n k_{q}}\right|^{r} \sum_{k_{q}-1=q-1}^{k_{q}-1} E\left|X_{n k_{q}-1}\right|^{r} \ldots \sum_{k_{1}=1}^{k_{2}-1} E\left|X_{n k_{1}}\right|^{r} .
\end{gathered}
$$

Now using the assumption (1.2) we get

$$
\begin{gathered}
\sum_{n=1}^{\infty} n^{\alpha r-2-\beta \delta} \sum_{j=1}^{\infty} j^{\delta-q r \gamma \alpha} \sum_{k_{q}=q}^{J} E\left|X_{n k_{q}}\right|^{r} \sum_{k_{q-1}=q-1}^{k_{q}-1} E\left|X_{n k_{q-1}}\right|^{r} \ldots \sum_{k_{1}=1}^{k_{2}-1} E\left|X_{n k_{1}}\right|^{r} \\
\leq \sum_{n=1}^{\infty} n^{\alpha r-2-\beta \delta} E|\xi|^{r} \sum_{j=1}^{\infty} j^{\delta-q r \gamma \alpha+1} \sum_{k_{q}=q}^{J} E\left|X_{n k_{q}}\right|^{r} \sum_{k_{q-1}=q-1}^{k_{q}-1} E\left|X_{n k_{q-1}}\right|^{r} \ldots \sum_{k_{1}=2}^{k_{3}-1} E\left|X_{n k_{2}}\right|^{r}
\end{gathered}
$$




$$
\leq \sum_{n=1}^{\infty} n^{\alpha r-2-\beta \delta}\left(E|\xi|^{r}\right)^{q} \sum_{j=1}^{\infty} j^{\delta+q(1-r \gamma \alpha)}<\infty
$$

as $\delta>\frac{\alpha r-1}{\beta}, \gamma>\frac{1}{\alpha r}$ and $q>\frac{1+\delta}{\gamma \alpha r-1}$.

To prove (2.5) for $i=3$ we write

$$
Y_{k j}^{n}=X_{n k} I\left[\left|X_{n k}\right|<j^{\gamma \alpha}\right]-E X_{n k} I\left[\left|X_{n k}\right|<j^{\gamma \alpha}\right],
$$

$1 \leq k \leq j, j \geq 1$ and $n \geq 1$.

Then we see that

$$
\begin{gathered}
\sum_{n=1}^{\infty} n^{\alpha r-2} P\left[B_{n}^{(3)} \cap\left[\nu_{n} \geq n^{\beta}\right]\right] \\
\leq \sum_{n=1}^{\infty} n^{\alpha r-2} \sum_{j \geq\left[n^{\beta}\right]} P\left[\left|\sum_{k \leq j} X_{n k} I\left[\left|X_{n k}\right|<j^{\gamma \alpha}\right]\right| \geq \frac{\varepsilon j^{\alpha}}{q}, \nu_{n}=j\right] \\
\leq \operatorname{const} \sum_{n=1}^{\infty} n^{\alpha r-2} \sum_{j \geq\left[n^{\beta}\right]} j^{-\alpha s} E\left|\sum_{k \leq j} X_{n k} I\left[\left|X_{n k}\right|<j^{\gamma \alpha}\right]\right|^{s} \\
\leq \text { const }\left[\sum_{n=1}^{\infty} n^{\alpha r-2} \sum_{J \geq\left[n^{\beta}\right]} j^{-\alpha s} E\left|\sum_{k \leq j} Y_{k j}^{n}\right|^{s}+\sum_{n=1}^{\infty} n^{\alpha r-2} \sum_{\jmath \geq\left[n^{\beta}\right]} j^{-\alpha s}\left|\sum_{k \leq j} E X_{n k} I\left[\left|X_{n k}\right|<j^{\gamma \alpha}\right]\right|^{s}\right]
\end{gathered}
$$

for every $s>0$ and a positive constant $c$.

We note that the second term in the last inequality is finite as

$$
\begin{gathered}
\sum_{n=1}^{\infty} n^{\alpha r-2} \sum_{\jmath \geq\left[n^{\beta}\right]} j^{-\alpha s}\left|\sum_{k \leq \jmath} E X_{n k} I\left[\left|X_{n k}\right|<j^{\gamma \alpha}\right]\right|^{s} \\
\leq \sum_{n=1}^{\infty} n^{\alpha r-2} \sum_{\jmath \geq\left[n^{\beta}\right]} j^{-\alpha s}\left(\sum_{k \leq j} \frac{E\left|X_{n k}\right|^{r} I\left[\left|X_{n k}\right| \geq j^{\gamma \alpha}\right]}{\left(j^{\gamma \alpha}\right)^{r-1}}\right)^{s} \\
\leq \sum_{n=1}^{\infty} n^{\alpha r-2} \sum_{j \geq\left[n^{\beta}\right]} j^{-\alpha s-\gamma \alpha(r-1) s}\left(\sum_{k \leq j} E\left|X_{n k}\right|^{r}\right)^{s} \\
\leq \text { const } \sum_{j=1}^{\infty} j^{-\alpha s+c-\gamma \alpha(r-1) s+s}\left(E|\xi|^{r}\right)^{s}=\operatorname{const}\left(E|\xi|^{r}\right)^{s} \sum_{j=1}^{\infty} j^{c-s(\alpha+\alpha r \gamma-\gamma \alpha-1)}<\infty
\end{gathered}
$$

for $s>\frac{c}{\alpha(1-\gamma)+\gamma \alpha r-1}$.

Now we can write

$$
\begin{gathered}
E\left|\sum_{k \leq j} Y_{k j}^{n}\right|^{s}=\int_{0}^{\infty} z^{s-1} P\left[\left|\sum_{k \leq j} Y_{k j}^{n}\right| \geq z\right] d z \\
=\int_{0}^{j \gamma \alpha} z^{s-1} P\left[\left|\sum_{k \leq j} Y_{k j}^{n}\right| \geq z\right] d z+\int_{j \vee \alpha}^{\infty} z^{s-1} P\left[\left|\sum_{k \leq j} Y_{k j}^{n}\right| \geq z\right] d z \\
\leq j^{\gamma \alpha s}+\int_{j \vee \alpha}^{\infty} z^{s-1} P\left[\left|\sum_{k \leq j} Y_{k j}^{n}\right| \geq z\right] d z .
\end{gathered}
$$

But the Fuk-Nagaev inequality (cf. Fuk and Nagaev [12]):

$$
P\left[\left|\sum_{i=1}^{n} X_{i}\right| \geq x\right]
$$




$$
\leq 2\left(\sum_{\imath=1}^{n} P\left[\left|X_{\imath}\right| \geq \eta x\right]+\frac{1}{(\eta x)^{t}} \sum_{\imath=1}^{n} \int_{0}^{\eta x}|u|^{t} d F_{X_{1}}(u)+\exp \left(-\frac{(1-\eta)^{2} x^{2}}{2 e^{t} \sum_{\imath=1}^{n} E X_{\imath}^{2}}\right)\right),
$$

where $t \geq 2, \eta=\frac{t}{t+2}$, allows us to show that

$$
\begin{gathered}
\int_{\jmath^{\gamma \alpha}}^{\infty} z^{s-1} P\left[\left|\sum_{k \leq \jmath} Y_{k \jmath}^{n}\right| \geq z\right] d z \\
\leq 2\left(\sum_{k=1}^{j} \int_{\jmath^{\gamma \alpha}}^{\infty} z^{s-1} P\left[\left|Y_{k \jmath}^{n}\right| \geq \eta z\right] d z+\frac{2}{\eta^{t}} \sum_{k=1}^{j} \int_{\jmath^{\gamma \alpha}}^{\infty} z^{s-t-1} \int_{0}^{\eta z}|u|^{t} d F_{Y_{k}^{n}}(u)\right. \\
\left.+\int_{\jmath^{\gamma \alpha}}^{\infty} z^{s-1} \exp \left(-\frac{(1-\eta)^{2} z^{2}}{2 e^{t} \sum_{k=1}^{j} E\left(Y_{k \jmath}^{n}\right)^{2}}\right) d z\right)
\end{gathered}
$$

Now we see that

$$
\begin{gathered}
\sum_{n=1}^{\infty} n^{\alpha r-2} \sum_{\jmath \geq\left[n^{\beta}\right]} j^{-\alpha s} \sum_{k \leq \jmath} \int_{j^{\gamma \alpha}}^{\infty} z^{s-1} P\left[\left|Y_{k j}^{n}\right| \geq \eta z\right] d z \\
=\left(\frac{1}{\eta}\right)^{s-1} \sum_{n=1}^{\infty} n^{\alpha r-2} \sum_{j \geq\left[n^{\beta}\right]} j^{-\alpha s} \sum_{k=1}^{J} E\left|Y_{k j}^{n}\right|^{s} \leq \mathrm{const} \sum_{j=1}^{\infty} j^{-\alpha s+c} j\left(j^{\gamma \alpha}\right)^{s}<\infty
\end{gathered}
$$

for $s>\frac{c+2}{\alpha(1-\gamma)}$.

Moreover, using the assumption on a regular cover (cf. Definition 1.2), we have

$$
\begin{gathered}
\sum_{n=1}^{\infty} n^{\alpha r-2} \sum_{J \geq\left[n^{\beta}\right]} j^{-\alpha s} \sum_{k=1}^{J} \int_{j^{r \alpha}}^{\infty} z^{s-t-1}\left(\int_{0}^{\eta z}|u|^{t} d F_{Y_{k j}^{n}}(u)\right) d z \\
\sum_{n=1}^{\infty} n^{\alpha r-2} \sum_{j \geq\left[n^{\beta}\right]} j^{-\alpha s} \sum_{k=1}^{J} \int_{j^{r \alpha}}^{\infty} z^{s-t-1}\left(E\left|Y_{k j}^{n}\right|^{t} I\left[\left|Y_{k j}^{n}\right|<z\right]\right) d z \\
\leq \text { const } \sum_{n=1}^{\infty} n^{\alpha r-2} \sum_{j \geq\left[n^{\beta}\right]} j^{-\alpha s} \sum_{k=1}^{J} E\left|X_{n k}\right|^{t} I\left[\left|X_{n k}\right|<j^{\gamma \alpha}\right] j^{\gamma \alpha(s-t)} \\
\leq \text { const } \sum_{n=1}^{\infty} n^{\alpha r-2} \sum_{j \geq\left[n^{\beta}\right]} j^{-\alpha s+\gamma \alpha(s-r)} \sum_{k=1}^{J} E\left|X_{n k}\right|^{r} \\
\leq \operatorname{const} E|\xi|^{r} \sum_{j=1}^{\infty} j^{-\alpha s+c+\gamma \alpha(s-r)+1}<\infty
\end{gathered}
$$

Moreover, using the assumption on a regular cover (cf. Definition 1.2), we have

for $s>\frac{c+2-\gamma \alpha r}{r(1-\alpha)}$.

Further on, we note that

$$
\begin{gathered}
\sum_{n=1}^{\infty} n^{\alpha r-2} \sum_{j \geq\left[n^{\beta}\right]} j^{-\alpha s} \int_{j^{\gamma \alpha}}^{\infty} z^{s-1} \exp \left(-\frac{(1-\eta)^{2} z^{2}}{2 e^{t} \sum_{k=1}^{j} E\left(Y_{k J}^{n}\right)^{2}}\right) d z \\
\leq \text { const } \sum_{n=1}^{\infty} n^{\alpha r-2} \sum_{j \geq\left[n^{\beta}\right]} j^{-\alpha s}\left(\sum_{k=1}^{j} E\left(Y_{k j}^{n}\right)^{2}\right)^{s / 2} \int_{0}^{\infty} y^{s / 2-1} e^{-y} d y \\
\leq \text { const } \sum_{n=1}^{\infty} n^{\alpha r-2} \sum_{j \geq\left[n^{\beta}\right]} j^{-\alpha s}\left(\sum_{k=1}^{J} E\left(Y_{k \jmath}^{n}\right)^{2}\right)^{s / 2} .
\end{gathered}
$$


Assume now that $r \geq 2$. Then we have

$$
\begin{gathered}
\sum_{n=1}^{\infty} n^{\alpha r-2} \sum_{j \geq\left[n^{\mathcal{\beta}}\right]} j^{-\alpha s}\left(\sum_{k=1}^{j} E\left(Y_{k \jmath}^{n}\right)^{2}\right)^{s / 2} \leq \mathrm{const} \sum_{n=1}^{\infty} n^{\alpha r-2} \sum_{j \geq\left[n^{\beta}\right]} j^{-\alpha s}\left(j E|\xi|^{2}\right)^{s / 2} \\
\leq \text { const } \sum_{j=1}^{\infty} j^{-\alpha s+c+s / 2}<\infty
\end{gathered}
$$

for $s>\frac{c+1}{\alpha-1 / 2}$.

Similarly it can be proved that for $r<2$

$$
\sum_{n=1}^{\infty} n^{\alpha r-2} \sum_{\jmath \geq\left[n^{\beta}\right]} j^{-\alpha s}\left(\sum_{k=1}^{\jmath} E Y_{k \jmath}^{2}\right)^{s / 2} \leq \text { const } \sum_{\jmath=1}^{\infty} j^{-s[\alpha-1 / 2-\gamma \alpha(2-r) / 2]+c}<\infty
$$

whenever $s>\frac{c+1}{\alpha-1 / 2+\gamma \alpha(2-r) / 2}$ and $\gamma$ is such that $\gamma<\frac{2 \alpha-1}{2-r}$.

Collecting the estimates $(2.7)-(2.14)$ we see that the series in (2.6) converges which completes the proof of $(2.1)$ for $\beta>0$.

But for the stronger requirement $\beta \geq 1$ we note that the condition ( 2.3) is fulfilled under the assumption $E\left|X_{n k}\right|^{r}<\infty, r \geq 1, k \geq 1, n \geq 1$.

Indeed, we see that

$$
\begin{aligned}
& \sum_{n=1}^{\infty} n^{\alpha r-2} P\left[\max _{k \leq \nu_{n}}\left|X_{n k}\right| \geq \varepsilon \nu_{n}^{\alpha}\right] \\
& \leq \sum_{n=1}^{\infty} n^{\alpha r-2} P\left[\nu_{n}<n^{\beta}\right]+\sum_{n=1}^{\infty} n^{\alpha r-2} P\left[\max _{k \leq \nu_{n}}\left|X_{n k}\right| \geq \varepsilon \nu_{n}^{\alpha}, \nu_{n} \geq n^{\beta}\right] \\
& \sum_{n=1}^{\infty} n^{\alpha r-2} P\left[\max _{k \leq \nu_{n}}\left|X_{n k}\right| \geq \varepsilon \nu_{n}^{\alpha}, \nu_{n} \geq n^{\beta}\right] \\
& \leq \text { const } \sum_{m=1}^{\infty}\left(2^{m}\right)^{\alpha r-1} P\left[\max _{k \leq \nu_{2}^{m}}\left|X_{2^{m} k}\right| \geq \varepsilon \nu_{2^{m}}^{\alpha}, \nu_{2^{m}} \geq\left(2^{m}\right)^{\beta}\right] \\
& \leq \text { const } \sum_{m=1}^{\infty}\left(2^{m}\right)^{\alpha r-1} \sum_{j=m}^{\infty} P\left[\max _{k \leq \nu^{m}}\left|X_{2^{m}}\right| \geq \varepsilon \nu_{2^{m}}^{\alpha},\left(2^{\jmath}\right)^{\beta} \leq \nu_{2^{m}}<\left(2^{j+1}\right)^{\beta}\right] \\
& \leq \text { const } \sum_{m=1}^{\infty}\left(2^{m}\right)^{\alpha r-1} \sum_{j=m}^{\infty} P\left[\max _{k \leq\left(2^{j+1}\right)^{\beta}}\left|X_{2^{m}}\right| \geq \varepsilon\left(2^{j}\right)^{\alpha \beta}\right] \\
& \leq \text { const } \sum_{m=1}^{\infty} P\left[\max _{k \leq\left(2^{m+1}\right) \rho}\left|X_{2^{m} k}\right| \geq \varepsilon\left(2^{m}\right)^{\alpha \beta}\right] \sum_{k=1}^{m}\left(2^{k}\right)^{\alpha r-1} \\
& \leq \text { const } \sum_{m=1}^{\infty}\left(2^{m}\right)^{\alpha r-1} P\left[\max _{k \leq\left(2^{m+1}\right)^{\beta}}\left|X_{2^{m} k}\right| \geq \varepsilon\left(2^{m}\right)^{\alpha \beta}\right] \\
& \leq \text { const } \sum_{m=1}^{\infty}\left(2^{m}\right)^{\alpha r-1} \sum_{k \leq\left(2^{m+1}\right)^{\beta}} P\left[\left|X_{2^{m} k}\right| \geq \varepsilon\left(2^{m}\right)^{\alpha \beta}\right] \\
& \leq \text { const } \sum_{m=1}^{\infty} \sum_{k \leq\left(2^{m+1}\right)^{\beta}} \frac{E\left|X_{2^{m} k}\right|^{r}}{\left(2^{m}\right)^{\alpha \beta r}} \\
& =\operatorname{const} E|\xi|^{r} \sum_{m=1}^{\infty}\left(2^{m}\right)^{\alpha r-1-\beta(\alpha r-1)}<\infty
\end{aligned}
$$


for $\beta \geq 1$, which gives (2.3) and ends the proof of Theorem 2.1.

Now we note that the condition $(2.3)(0<\beta<1)$ is fulfilled under a stronger moment condition than that of Theorem 2.1.

COROLLARY. Let $\left\{X_{n k}, n \geq 1, k \geq 1\right\}$ be an array of rowwise independent random variables such that $X_{n 1}, X_{n 2}, \ldots, X_{n k}, n \geq 1, k \geq 1$, form an independent regular cover of a random variable $\xi$, and assume that $E X_{n k}=0, E\left|X_{n k}\right|^{\frac{\alpha r-1+\beta}{\alpha \beta}}<\infty, n \geq 1, k \geq 1, E \xi=0$, and $E|\xi|^{\frac{a r-1+\beta}{\alpha \beta}}<\infty$ for $r \geq 1, \alpha>1 / 2, \alpha r>1,0<\beta<1$.

If $\left\{\nu_{n}, n \geq 1\right\}$ is a sequence of positive integer-valued random variables such that

$$
\sum_{n=1}^{\infty} n^{\alpha r-2} P\left[\nu_{n}<n^{\beta}\right]<\infty,
$$

then for any given $\varepsilon>0$

$$
\sum_{n=1}^{\infty} P\left[\left|S_{\nu_{n}}\right| \geq \varepsilon \nu_{n}^{\alpha}\right]<\infty .
$$

PROOF. It is enough to see that under the considered case the condition (2.3) is satisfied.

Since

$$
\begin{gathered}
\sum_{n=1}^{\infty} n^{\alpha r-2} P\left[\max _{k \leq \nu_{n}}\left|X_{n k}\right| \geq \varepsilon \nu_{n}^{\alpha}\right] \\
\leq \sum_{n=1}^{\infty} n^{\alpha r-2} P\left[\nu_{n}<n^{\beta}\right]+\sum_{n=1}^{\infty} n^{\alpha r-2} P\left[\max _{k \leq \nu_{n}}\left|X_{n k}\right| \geq \varepsilon \nu_{n}^{\alpha}, \nu_{n} \geq n^{\beta}\right],
\end{gathered}
$$

then we need only to note that

$$
\begin{gathered}
\sum_{n=1}^{\infty} n^{\alpha r-2} P\left[\max _{k \leq \nu_{n}}\left|X_{n k}\right| \geq \varepsilon \nu_{n}^{\alpha}, \nu_{n} \geq n^{\beta}\right] \\
\leq \text { const } \sum_{m=1}^{\infty}\left(2^{m}\right)^{\alpha r-1} P\left[\max _{k \leq \nu_{2} m}\left|X_{2^{m} k}\right| \geq \varepsilon \nu_{2^{m}}^{\alpha}, \nu_{2^{m}} \geq\left(2^{m}\right)^{\beta}\right] \\
\leq \text { const } \sum_{m=1}^{\infty}\left(2^{m}\right)^{\alpha r-1} \sum_{j=m}^{\infty} P\left[\max _{k \leq \nu_{2}^{m}}\left|X_{2^{m} k}\right| \geq \varepsilon \nu_{2^{m}}^{\alpha},\left(2^{j}\right)^{\beta} \leq \nu_{2^{m}}<\left(2^{j+1}\right)^{\beta}\right] \\
\leq \text { const } \sum_{m=1}^{\infty}\left(2^{m}\right)^{\alpha r-1} \sum_{j=m}^{\infty} P\left[\max _{k \leq\left(2^{j+1}\right)^{\beta}}\left|X_{2^{m} k}\right| \geq \varepsilon\left(2^{j}\right)^{\alpha \beta}\right] \\
\leq \text { const } \sum_{m=1}^{\infty} P\left[\max _{k \leq\left(2^{m+1}\right)^{\beta}}\left|X_{2^{m} k}\right| \geq \varepsilon\left(2^{m}\right)^{\alpha \beta}\right] \sum_{k=1}^{m}\left(2^{k}\right)^{\alpha r-1} \\
\leq \text { const } \sum_{m=1}^{\infty}\left(2^{m}\right)^{\alpha r-1} P\left[\max _{k \leq\left(2^{m+1}\right)^{\beta}}\left|X_{2^{m} k}\right| \geq \varepsilon\left(2^{m}\right)^{\alpha \beta}\right] \\
\leq \text { const } \sum_{m=1}^{\infty}\left(2^{m}\right)^{\alpha r-1} \sum_{k \leq\left(2^{m+1}\right)^{\beta}} P\left[\left|X_{2^{m} k}\right| \geq \varepsilon\left(2^{m}\right)^{\alpha \beta}\right] \\
\leq \text { const } \sum_{m=1}^{\infty}\left(2^{m}\right)^{\alpha r-1+\beta} P\left[|\xi|^{\frac{1}{\alpha \beta}} \geq \varepsilon\left(2^{m}\right)\right] \leq \operatorname{const} E|\xi|^{\frac{\alpha r-1+\beta}{\alpha \beta}}<\infty .
\end{gathered}
$$

Note that the moment condition of Corollary is close to optimal which shows the following statement. 
THEOREM 2.2. Let $\left\{X_{n k}, n \geq 1, k \geq 1\right\}$ be an array of rowwise independent random variables such that $X_{n 1}, X_{n 2}, \ldots, X_{n k}, n \geq 1, k \geq 1$, form an independent regular cover of a random variable $\xi$, and assume that $E X_{n k}=0$.

Then for $r \geq 1, \alpha>1 / 2, \alpha r>1, \beta>0$, the convergence of the series

$$
\sum_{n=1}^{\infty} n^{\alpha r-2} P\left[\left|S_{\left[n^{\beta}\right]}\right| \geq \varepsilon n^{\alpha \beta}\right]<\infty
$$

implies $E|\xi|^{\frac{\alpha r-1+\beta}{\alpha \beta}}<\infty$.

PROOF. Let $\mu_{n}$ be a median of $S_{n}$, i.e. $\mu_{n}=\left\{t: P\left[S_{n}<t\right] \geq 1 / 2\right\}$. By the standard symmetrization inequalities (cf. Loève [13]) we have

$$
\begin{gathered}
P\left[\left|S_{\left[n^{\beta}\right]}\right| \geq \varepsilon n^{\alpha \beta}\right] \\
\geq \frac{1}{2} P\left[\left|S_{\left[n^{\beta}\right]}^{s}\right| \geq 2 \varepsilon n^{\alpha \beta}\right] \geq \frac{1}{4} P\left[\left|S_{\left[n^{\beta}\right]}-\mu_{\left[n^{\beta}\right]}\right| \geq 2 \varepsilon n^{\alpha \beta}\right] \\
\geq \frac{1}{4} P\left[S_{\left[n^{\beta}\right]}-\mu_{\left[n^{\beta}\right]} \geq 2 \varepsilon n^{\alpha \beta}\right],
\end{gathered}
$$

which by $(2.15)$ gives

$$
\sum_{n=1}^{\infty} n^{\alpha r-2} P\left[S_{\left[n^{\beta}\right]}-\mu_{\left[n^{\beta}\right]} \geq 2 \varepsilon n^{\alpha \beta}\right]<\infty .
$$

We note that $\tau_{n}=\sup \left\{\tau: P[\xi \geq \tau] \geq \frac{1}{4 n^{\beta}}\right\}$. We note that $\tau_{n} \geq \tau_{n-1}$, and

$$
P\left[\xi \geq \tau_{n}\right] \geq \frac{1}{4 n^{\beta}}, P\left[\xi \leq \tau_{n}\right] \geq 1-\frac{1}{4 n^{\beta}} .
$$

If the $\tau_{n}$ are all negative then $P[\xi<0]=1$ so $E\left(\xi^{+}\right)^{\frac{\alpha r-1+\beta}{\alpha \beta}}=0<\infty$. Thus, assume that for $n$ sufficiently large we have $\tau_{n} \geq 0$. Moreover, we note that by (2.17)

$$
\begin{gathered}
P\left[X_{n k}>\tau_{n}\right] \leq P\left[X_{n 1}>\tau_{1}\right]+\ldots+P\left[X_{n\left[n^{\beta}\right]}>\tau_{n}\right] \\
\leq n^{\beta} P\left[\xi>\tau_{n}\right]=n^{\beta}\left(1-P\left[\xi \leq \tau_{n}\right]\right) \leq \frac{1}{4}
\end{gathered}
$$

Furthemore, for $k \in\left\{1, \ldots,\left[n^{\beta}\right]\right\}$ define $\left\{\rho_{n k}, 1 \leq k \leq\left[n^{\beta}\right]\right\}$ with

$$
\rho_{n k}=\sup \left\{\rho: P\left[S_{\left[n^{\beta}\right]}-X_{n k} \geq \rho\right] \geq \frac{1}{3}\right\}
$$

Then we have

$$
P\left[S_{\left[n^{\beta}\right]}-X_{n k} \geq \rho_{n k}\right] \geq \frac{1}{3}, P\left[S_{\left[n^{\beta}\right]}-X_{n k} \leq \rho_{n k}\right] \geq \frac{2}{3} .
$$

Using the independence $S_{\left[n^{\beta}\right]}-X_{n k}$ and $X_{n k},(2.18)$ and (2.19) we get

$$
\begin{gathered}
P\left[S_{\left[n^{\beta}\right]} \leq \tau_{n}+\rho_{n k}\right] \geq P\left[X_{n k} \leq \tau_{n}, S_{\left[n^{\beta}\right]}-X_{n k} \leq \rho_{n k}\right] \\
=P\left[X_{n k} \leq \tau_{n}\right] P\left[S_{\left[n^{\beta}\right]}-X_{n k} \leq \rho_{n k}\right] \\
=\left(1-P\left[X_{n k}>\tau_{n}\right]\right) P\left[S_{\left[n^{\beta}\right]}-X_{n k} \leq \rho_{n k}\right] \geq \frac{1}{2} .
\end{gathered}
$$

Now using

$$
T_{n k}:=\left[X_{n k}>2 \varepsilon n^{\alpha \beta}+\tau_{n}\right], R_{n k}:=\left[S_{\left[n^{\beta}\right]}-X_{n k} \geq \rho_{n k}\right]
$$


we see that

$$
\begin{gathered}
P\left[S_{\left[n^{\beta}\right]} \geq 2 \varepsilon n^{\alpha \beta}+\mu_{\left[n^{\beta}\right]}\right] \\
\geq P\left[S_{\left[n^{\beta}\right]}>2 \varepsilon n^{\alpha \beta}+\tau_{n}+\rho_{n k}\right] \geq P\left[\bigcup_{k=1}^{\left[n^{\beta}\right]}\left(T_{n k} \cap R_{n k}\right)\right] \\
=\sum_{k=1}^{\left[n^{\beta}\right]} P\left[\left(T_{n 1} \cap R_{n 1}\right)^{c} \cap \ldots \cap\left(T_{n k-1} \cap R_{n k-1}\right)^{c} \cap\left(T_{n k} \cap R_{n k}\right)\right] \\
\geq \sum_{k=1}^{\left[n^{\beta}\right]} P\left[T_{n 1}^{c} \cap \ldots \cap T_{n k-1}^{c} \cap T_{n k} \cap R_{n k}\right] \\
\geq \sum_{k=1}^{\left[n^{\beta}\right]}\left\{P\left[T_{n k} \cap R_{n k}\right]-P\left[\left(T_{n 1} \cup \ldots \cup T_{n k-1}\right) \cap R_{n k}\right]\right\} \\
\geq \sum_{k=1}^{\left[n^{\beta}\right]} P\left[T_{n k}\right]\left\{P\left[R_{n k}\right]-\sum_{k=1}^{\left[n^{\beta}\right]} P\left[T_{n k}\right]\right\}
\end{gathered}
$$

Having $\tau_{n} \geq 0$ for sufficiently large $n$ we get

$$
\begin{gathered}
\sum_{k=1}^{\left[n^{\beta}\right]} P\left[T_{n k}\right]=\sum_{k=1}^{\left[n^{\beta}\right]} P\left[X_{n k} \geq 2 \varepsilon n^{\alpha \beta}+\tau_{n}\right] \\
-\infty n^{\beta} P\left[\xi>2 \varepsilon n^{\alpha \beta}+\tau_{n}\right]=n^{\beta}\left(1-P\left[\xi \leq 2 \varepsilon n^{\alpha \beta}+\tau_{n}\right]\right) \leq \frac{1}{4},
\end{gathered}
$$

where we have used the covering identity (1.1) as well as (2.17).

Thus, (2.20) implies that

$$
P\left[S_{\left[n^{\beta}\right]} \geq 2 \varepsilon n^{\alpha \beta}+\mu_{\left[n^{\beta}\right]}\right] \geq \frac{1}{12}\left[n^{\beta}\right] P\left[\xi>2 \varepsilon n^{\alpha \beta}+\tau_{n}\right]
$$

for $n$ sufficiently large.

Hence, by (2.16) we conclude that

$$
\sum_{n=1}^{\infty} n^{\alpha r-2+\beta} P\left[\xi>2 \varepsilon n^{\alpha \beta}+\tau_{n}\right]<\infty
$$

which is equivalent to

$$
\sum_{m=1}^{\infty}\left(2^{m}\right)^{\alpha r-1+\beta} P\left[\xi>2 \varepsilon\left(2^{m}\right)^{\alpha \beta}+\tau_{2^{m}}\right]<\infty
$$

Similarly as in Pruss [11] (cf. Lemma 4) we can show that for $m$ sufficiently large we have

$$
\tau_{2^{m+1}} \leq 2^{m} \varepsilon+\tau_{2^{m}}
$$

Assume that $M$ is a positive integer number such that

$$
\tau_{2^{m+1}} \leq 2 \varepsilon\left(2^{m}\right)^{\alpha \beta}+\tau_{2^{m}} \text { for } \quad m \geq M .
$$

Iterating this inequality for $m \geq M$ we obtain

$$
\tau_{2^{m}}<2 \varepsilon\left(2^{m}\right)^{\alpha \beta}+\tau_{2^{M}}
$$


which gives $2 \varepsilon\left(2^{m}\right)^{\alpha \beta}+\tau_{2^{m}}<4 \varepsilon\left(2^{m}\right)^{\alpha \beta}+\tau_{2^{M}}$.

Therefore, using (2.21), we have

$$
\sum_{m=1}^{\infty}\left(2^{m}\right)^{\alpha r-1+\beta} P\left[\xi>4 \varepsilon\left(2^{m}\right)^{\alpha \beta}+\tau_{2} M\right]<\infty
$$

which proves that

$$
\begin{gathered}
\infty>\sum_{n=1}^{\infty} n^{\alpha r-2+\beta} P\left[\xi>4 \varepsilon n^{\alpha \beta}+\tau_{2} M\right] \\
\geq \sum_{n=1}^{\infty} n^{\alpha r-2+\beta} P\left[\xi>\left(4 \varepsilon+\tau_{2} M\right) n^{\alpha \beta}\right] \geq \operatorname{const} E\left(\xi^{+}\right)^{\frac{\alpha r-1+\beta}{\alpha \beta}} .
\end{gathered}
$$

Similarly one can show that $E\left(\xi^{-}\right)^{\frac{\alpha r-1+\beta}{\alpha \beta}}<\infty$, which completes the proof of Theorem 2.2.

ACKNOWLEDGEMENT. We are very grateful to the referee for his helpful comments allowing us to improve the previous version of the paper.

\section{REFERENCES}

1. HSU, P.L. and ROBBINS, H., "Complete Convergence and the Law of Large Numbers", Proc. Nat. Acad. Sci. U.S.A. 33, (1947) 25-31.

2. ERDÖS, P., "On a Theorem of Hsu and Robbins", Ann. Math. Statist. 20, (1949) 286-291.

3. GUT, A., "Complete Convergence", technical report.

4. SZYNAL, D., "On Almost Complete Convergence for the Sums of a Random Numbers of Independent Random Variables", Bull.Acad. Polon. Math. 20 (1972) 571-574.

5. GUT, A., "Complete Convergence and Convergence Rates for Randomly Indexed Partial Sums with an Application to Some First Passage Times", Acta Math. Hung. 42 (1983) 225-232.

6. ZHIDONG, B. and CHUN, S., "The Complete Convergence for Partial Sums of I.I.D. Random Variables", Sci. Sinica Ser. A 28 (1985) 1261-1277.

7. ADLER, A., "On Complete Convergence of the Sum of a Ramdom Number of Random Variables", Calcutta Statist. Assoc. Bull., 37 (1988) 161-169.

8. KLESOV, O.I., "The Rate of Convergence of Sums of Random Number of Random Variables", Theor. Probability and Math. Statist. 39 (1989) 75-81.

9. KUCZMASZEWSKA, A. and SZYNAL, D., "On the Complete Convergence for Randomly Indexed Partial Sums and the Limit Behaviour of a Sequence of Quantiles", J. Statist. Plann. Inference. 30 (1992) 371-383.

10. KUCZMASZEWSKA, A. and SZYNAL, D., "On Complete Convergence in a Banach Space", Internat, J. Math. \& Math. Sci. 17 (1994) 1-14.

11. PRUSS, A.R., "Randomly Sampled Riemann Sums and Complete Convergence in the Law of Large Numbers for a Case without Identical Distribution", Proc. Amer. Math. Soc. 124 (1996) 919-929

12. FUK, D. Kh. and NAGAEV, S. V., "Probabilistic Inequalities for Sums of Independent Random Variables", Theory Probab. Appl. 16 (1971) 660-675.

13. LOÈVE, M., Probability Theory. 4rd ed. Vol 1 van Nostrand Princeton, (1977). 


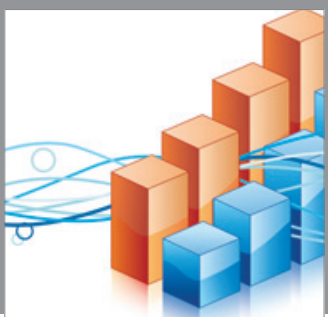

Advances in

Operations Research

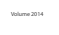

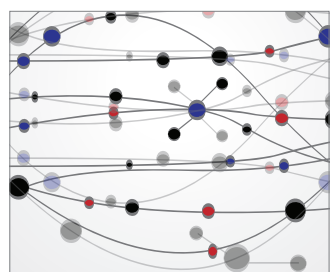

\section{The Scientific} World Journal
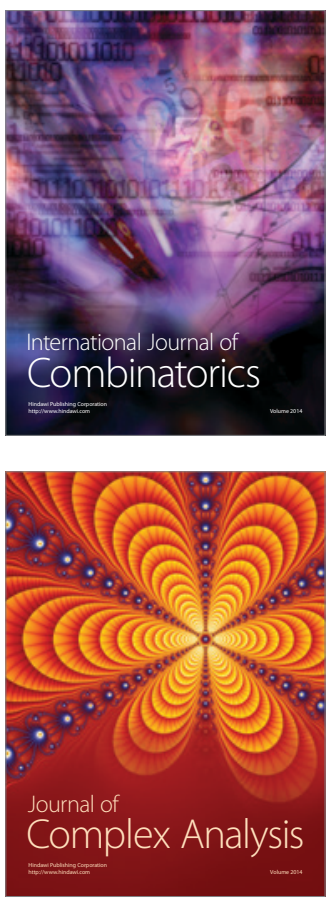

International Journal of

Mathematics and

Mathematical

Sciences
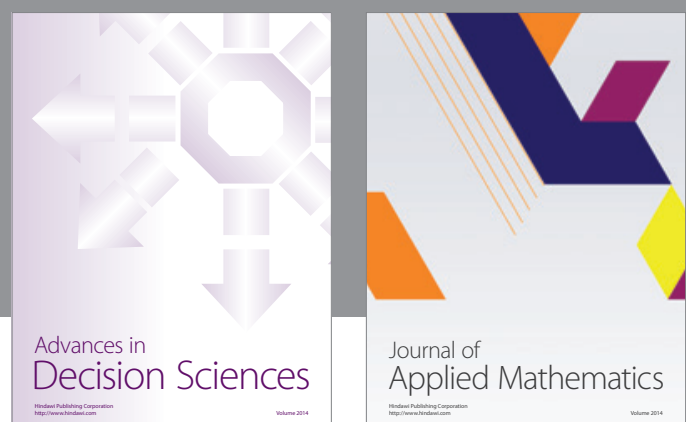

Journal of

Applied Mathematics
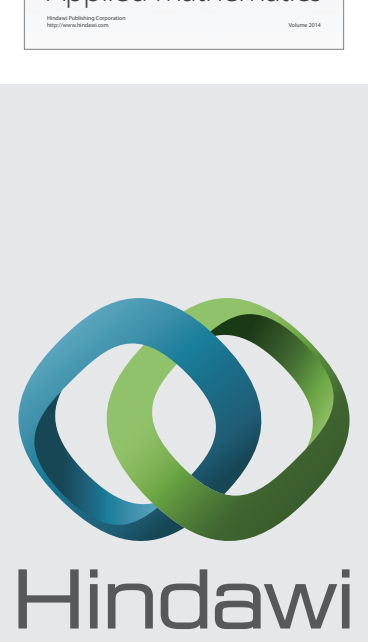

Submit your manuscripts at http://www.hindawi.com
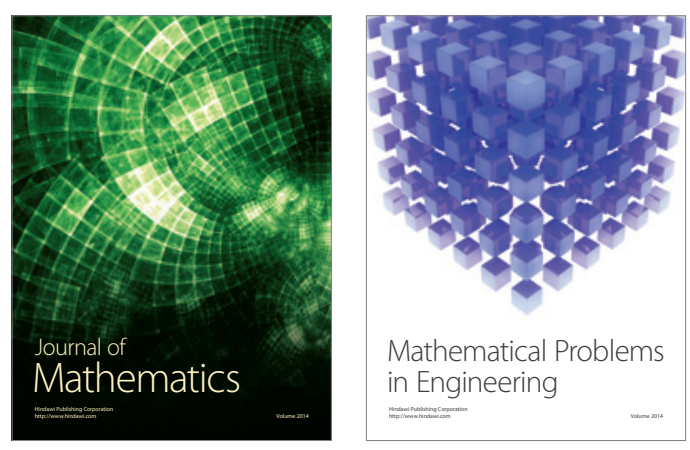

Mathematical Problems in Engineering
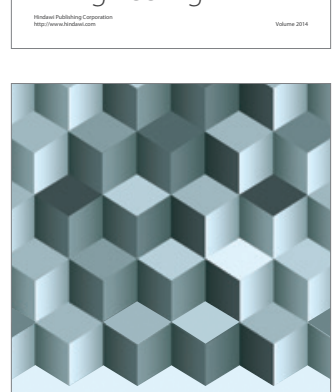

Journal of

Function Spaces
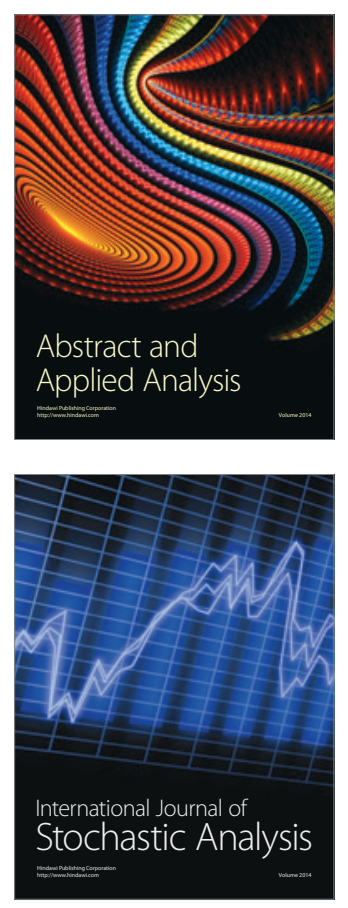

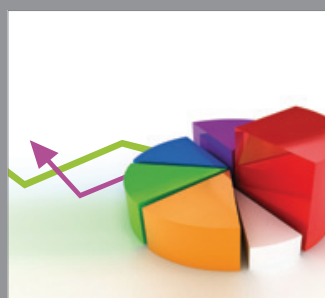

ournal of

Probability and Statistics

Promensencen
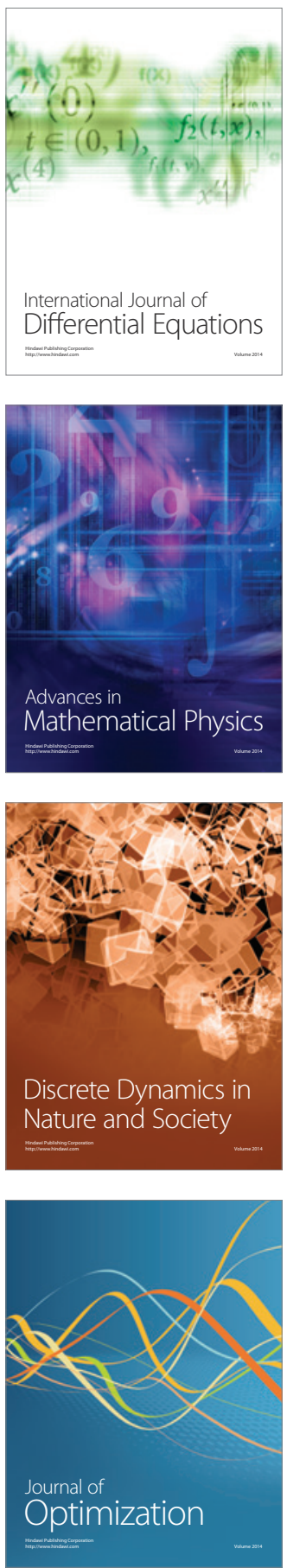